\title{
EXPOSIÇÃO OCUPACIONALA FLUIDOS BIOLÓGICOS EM ACIDENTES COM PERFUROCORTANTES NA EQUIPE DE ENFERMAGEM HOSPITALAR
}

José Aderval Aragão³, Leticia Moreira Fontes², Iapunira Catarina Sant'Anna Aragão³, Felipe Matheus Sant'Anna Aragão4, Francisco Prado Reis $^{4}$

Objetivo: Determinar a ocorrência de exposição ocupacional a fluidos biológicos em acidentes com perfurocortantes na equipe de enfermagem hospitalar. Métodos: Avaliou-se 747 profissionais, com base em questionários para acidentes de trabalho, mediante entrevista. Resultados: Dos 747 entrevistados, 53,9\% sofreram acidentes com perfurocortantes. O risco aumentado de sofrer acidentes relacionou-se com o sexo feminino, horário diurno de trabalho e profissões técnico e auxiliar de enfermagem. Grande parte dos acidentes ocorreu por reencape de agulhas e descarte inadequado dos materiais, por falta de atenção. Mais de um terço dos acidentados não comunicou o acidente. Conclusão: Os profissionais estudados mantêm posturas de risco que devem ser combatidas com políticas de educação em saúde, reforçando a importância da comunicação das ocorrências Descritores: Agentes biológicos; Acidentes ocupacionais; Ferimentos penetrantes; Equipe de enfermagem.

\section{OCCUPATIONAL EXPOSURE TO BIOLOGICAL FLUIDS IN ACCIDENTS WITH PROFILING ON THE HOSPITAL NURSING TEAM}

Objective: To determine the occurrence of occupational exposure to biological fluids in sharps injuries in the hospital nursing team. Methods: A total of 747 professionals were interviewed, based on questionnaires for work accidents. Results: 0 f the 747 interviewees, $53.9 \%$ suffered accidents by sharp instruments. The increased risk of accidents was related to the female sex, daytime work hours and nursing technician and assistant professions. A large part of the accidents occurred due to needle recapping and inadequate disposal of materials, due to lack of attention. More than a third of the victims did not report the accident. Conclusion: The studied professionals have attitudes of risk that must be tackled with policies of health education, reinforcing the importance of the communication of occurrences.

Descriptors: Biological factors; Accidents, occupational; Wounds, penetrating; Nursing, team.

\section{EXPOSICIÓN OCUPACIONAL A FLUIDOS BIOLÓGICOS EN ACCIDENTES COM PERFUROCORTANTES EN EL EQUIPO DE ENFERMERÍA HOSPITALARIA}

Objetivo: Exposición ocupacional a fluidos biológicos en accidentes con punzocortantes en el equipo de enfermería hospitalaria. Métodos: Se evaluaron 747 profesionales, con base en cuestionarios para accidentes de trabajo, mediante entrevista. Resultados: De los 747 entrevistados, 53,9\% sufrieron accidentes con punzocortantes. El riesgo aumentado de sufrir accidentes se relacionó con el sexo femenino, horario diurno de trabajo y profesiones técnicas y auxiliares de enfermería. Gran parte de los accidentes ocurrió por reencape de agujas y descarte inadecuado de los materiales, por falta de atención. Más de un tercio de los accidentes no comunicó el accidente. Conclusión: Los profesionales estudiados mantienen posturas de riesgo que deben ser combatidas con políticas de educación en salud, reforzando la importancia de la comunicación de las ocurrencias.

Descritores: Factores Biológicos; Accidentes de trabajo; Heridas penetrantes; Grupo de Enfermería. 


\section{INTRODUÇÃO}

Os acidentes ocupacionais são agravos de grande impacto à saúde pública, que podem levara consequências temporárias e até incapacidade permanente. Os acidentes que envolvem material biológico são especialmente preocupantes pela possibilidade de transmissão de doenças infectocontagiosas altamente incapacitantes, quando não letais, como AIDS e Hepatite ${ }^{(1)}$.

Os profissionais de enfermagem têm sido apontados como a classe mais vulnerável aos acidentes com material biológico. Isto possivelmente seria devido, sobretudo, por estarem em contato prolongado e constante com os pacientes, durante a realização de procedimentos envolvendo a manipulação de materiais perfurocortantes, responsáveis por grande parte dos acidentes ${ }^{(2,3)}$

Pesquisas realizadas no Brasil identificaram grande incidência de acidentes com material biológico ${ }^{(2,4)}$, assim como altos índices de subnotificação( ${ }^{(5)}$. Esta subnotificação tem ocorrido a despeito da existência de órgãos como a Comissão Interna de Prevenção de Acidentes (CIPA) e o Serviço Especializado em Engenharia de Segurança e Medicina do Trabalho (SESMT), ambos direcionados à prevenção de acidentes ocupacionais e doenças decorrentes do trabalho.

Desse modo dados epidemiológicos acerca deste tema tornam-se muito importantes para colaborar com o desenvolvimento de estratégias públicas, a educação em saúde e a prevenção desse tipo de agravo. O presente trabalho teve por objetivo determinar a ocorrência de exposição ocupacional a fluidos biológicos em acidentes com perfurocortantes na equipe de enfermagem hospitalar.

\section{METODOLOGIA}

\section{Tipo de estudo}

Trata-se de um estudo descritivo e exploratório, com desenho transversal

\section{Participantes da pesquisa}

Foram incluídos no estudo toda equipe de enfermagem (enfermeiros, auxiliares e técnicos de enfermagem) de dois hospitais da rede pública de Aracaju/SE no período de agosto de 2013 a fevereiro de 2014. Foram excluidos os profissionais que, no periodo da coleta, estavam em férias ou não desejaram fazer parte da pesquisa.

\section{Local de estudo}

O estudo foi desenvolvido em dois hospitais, um destinado ao atendimento de urgências e emergências de média e alta complexidade, recebendo demanda de todo o estado além de cidades de outros estados nordestinos, principalmente os da Bahia e de Alagoas. O outro destina-se à realização de cirurgias eletivas e atendimento referenciado de urgências.

\section{Coleta de dados}

A coleta dos dados ocorreu através da aplicação de um questionário previamente elaborado especificamente para este estudo com base no instrumento proposto pela National Institute for Occupational Safety and Health(NIOSH, 1999).

\section{Analise dos dados}

$O$ banco de dados foi construído em planilha Excel e posteriormente exportado para o programa SPSS 19.0 versão teste para a análise estatística. As variáveis numéricas foram descritas como média e desvio padrão. As variáveis categóricas foram descritas com frequências simples e porcentagem e, para as variáveis-desfecho, intervalo de confiança de 95\% (IC95\%). A estimativa do IC95\% foi realizada mediante a técnica de bootstrap, considerando-se 1000 re-amostragens com reposição de tamanho de 747. O nível de confiança foi 0,05 para erro e poder de 0,80 e os testes assumidos como bicaudais. Para análise dos fatores associados ao evento acidente foi utilizada a regressão logística, considerando-se para entrada no modelo $p=0,35$ e para permanência no mesmo p=0,05 e calculadas as razões de chances, ou odds ratio (OR) simples e ajustadas.

\section{Procedimentos éticos}

Depois de explicado os objetivos do estudo, todos os sujeitos eram convidados a participar da pesquisa, e solicitados a assinarem um Termo de Consentimento Livre e Esclarecido. O projeto foi submetido ao comitê de ética e pesquisa com seres humanos da Universidade Federal de Sergipe e aprovado sob o protocolo no: CAAE - 0131.0.107.000-10.

\section{RESULTADOS}

Dos 747 profissionais de enfermagem, componentes das equipes dos dois hospitais públicos pesquisados: 314 (42\%) eram auxiliares de enfermagem, 342 (45,8\%) técnicos de enfermagem e 91 (12,2\%) enfermeiros. A idade média foi de 38,5 \pm 9,3 anos, com a mínima de 20 e a máxima 66 anos de idade, sendo 591 (79,1\%) do sexo feminino e 156 (20,9\%) do masculino.

Os profissionais tinham em média 9,5 \pm 7,9 anos de tempo de serviço nas instituições, com mínimo de 1 mês e máximo de 39 anos. Dos entrevistados, 25\% tinham uma permanência de trabalho de até 2,8 anos, $50 \%$ até 6,3 anos e $25 \%$ mais do que 16,5 anos.

Quanto ao tempo de experiência profissional dos entrevistados: $25 \%$ tinham até 5,5 anos, $50 \%$ até 11 anos e $25 \%$ tinham mais de 20 anos de experiência profissional. A média foi de 12,6 \pm 8,8 anos, com mínimo de 1 mês e máximo 
de 43 anos.

Analizando a cobertura vacinal contra a hepatite $B$, foi encontrada uma alta porcentagem de indivíduos não imunizados, e também que ignoraram a pergunta. Estes individuos representaram quase um quinto do total dos profissionais (131; 17,5\%).

Afrequência de acidentes com materiais perfurocortantes foi de 53,9\% (403/747) com IC 95\% de 50,1 a 57,6\%. Mais de um terço dos entrevistados não notificou a ocorrência (144; 35,7\%). Dos 259, 121 (46\%) notificaram à CCIH (Comissão de Controle de Infecção Hospitalar), 96 (37,1\%) ao SESMT e 25 $(9,6 \%)$ aos chefes de setores, enquanto 17 não responderam qual foi o órgão notificado.

Dentre os fatores sócio-demográficos associados à ocorrência dos acidentes, a média de idade dos profissionais acidentados foi significativamente maior ( $p=0,029)$ em relação ao grupo dos não acidentados. Foi encontrada uma relação entre o gênero e os episódios de acidentes perfurocortantes, com uma maior frequência do sexo feminino no grupo dos acidentados ( $p<0,0001)$. Houve também associação entre o nível de escolaridade e a ocorrência de acidentes $(p=0,015)$, com um percentual menor entre os indivíduos de nível superior completo. A situação de estado civil não apresentou relação com a ocorrência de acidentes $(p=0,55)$ (Tabela 1$)$.

Tabela 1. Caracteristicas sócio-demograficas dos profissionais de enfermagem segundo a ocorrência de acidentes com materiais perfurocortantes $(\mathrm{N}=747)$

\begin{tabular}{|c|c|c|c|}
\hline VARIÄVETS & $\begin{array}{c}\text { Acideatadon } \\
\text { B-403 }\end{array}$ & $\begin{array}{c}\text { Nào acideatados } \\
\text { - } 344\end{array}$ & $p^{*}$ \\
\hline Idade' & $39.2 \pm 9.8$ & $37,7 \pm 9,9$ & 0,029 \\
\hline \multicolumn{4}{|l|}{$\operatorname{Sex} 0,0(96)$} \\
\hline Feminino & $351(87,1)$ & $240(69,3)$ & $<0.0001$ \\
\hline Mnseulins & $52(12,9)$ & $104(30,2)$ & \\
\hline \multicolumn{4}{|l|}{ Estado eivil, a(96) } \\
\hline Solneiro & $142(35,2)$ & $135(39,2)$ & 0,55 \\
\hline Casado & $157(46,4)$ & $156(45,3)$ & \\
\hline Divortiadss & $43(16,7)$ & $35(10.2)$ & \\
\hline Viuves & $6(1.5)$ & $5(1.5)$ & \\
\hline Outros & $25(6,2)$ & $13(3,5)$ & \\
\hline \multicolumn{4}{|l|}{ Grat de escelandade, n(*o) } \\
\hline Fasino méfio incompleto & $6(1,5)$ & $10(3,0)$ & 0,015 \\
\hline Easiso medfo completo & $271(67,2)$ & $2 ! 0(61,0)$ & \\
\hline Easiao seperios iasotaplete & $47(4,7)$ & $30(8.7)$ & \\
\hline Easino itperioe completo & $79(19,6)$ & $94(27.3)$ & \\
\hline
\end{tabular}

${ }^{1}$ Idade em anos expressa como média e desvio padrão; *comparação entre os grupos acidentados e não acidentados. Teste t de Student para dados independentes. Teste do quiquadrado ou exato de Fisher.
Em relação à distribuição por categoria profissional, houve associação significante $(p<0,0001)$, com frequência maior entre os auxiliares de enfermagem e menor entre os enfermeiros. Não foi encontrada relação entre os setores de trabalho no hospital e a ocorrência de acidentes com perfurocortantes $(p=0,069)$. A análise mostrou maior tempo de exercício profissional e de serviço no hospital entre os profissionais acidentados, quando comparados aos não acidentados, com diferença significativa ( $p=0,04$ e 0,006 respectivamente). Ademais, observou-se que o turno noturno de trabalho esteve associado com menor ocorrência de acidentes $(p=0,018)$ (Tabela 2$)$.

Tabela 2. Distribuição dos profissionais de enfermagem entrevistados conforme a categoria profissional, setor de trabalho no hospital, tempos de exercício na profissão e de serviço na instituição e turno noturno de trabalho (N=747)

\begin{tabular}{|c|c|c|c|}
\hline VARIAVES & $\begin{array}{c}\text { Acibestadon } \\
\text { ind }\end{array}$ & 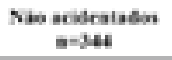 & $p^{*}$ \\
\hline \multicolumn{4}{|l|}{ Catrgarta pronuceal, a(4) } \\
\hline Enferavere & $22(5,5)$ & $\theta(x 6.1)$ & $\infty .0601$ \\
\hline Teraike de esferagpea & $394(46,7)$ & $154(64.5)$ & \\
\hline Awoise de tedionagie & $393(47,9)$ & (2t (a6) & \\
\hline \multicolumn{4}{|l|}{ Seser te enstallas $=(t)$ ) } \\
\hline Als cietrgika" & $108\{23,0\}$ & $35(25,9)$ & 0,000 \\
\hline Alss endicat? & $110(27,3)$ & $30(23,2)$ & \\
\hline CMi: & $19(4,7)$ & $8(1,7)$ & \\
\hline 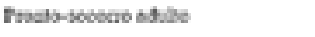 & $110(27,9)$ & $96(28.0)$ & \\
\hline uni & $32(7,9)$ & $3 s(100,2)$ & \\
\hline Als podurrics' & $29(7,2)$ & $35(11.0)$ & \\
\hline Terope de rurcirie as prefondot & $183=8,4$ & $12=9.2$ & 4.94 \\
\hline 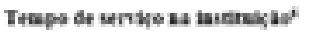 & $10.3=7.6$ & $2.7=8.2$ & 0,006 \\
\hline Tarses anteres, s(*4) & $16996,0\}$ & $153(6)$ & 0,035 \\
\hline
\end{tabular}

${ }^{1}$ Centro cirúrgico, enfermaria cirúrgica e clínica cirúrgica; ${ }^{2}$ Enfermaria médica, clínica médica e clínicas de especialidades; ${ }^{3}$ Centro de Material e Esterilização; 4Unidade de Terapia Intensiva;

${ }^{5}$ Inclui pronto-socorro infantil;

${ }^{6}$ Tempo em anos expresso como média e desvio padrão. *comparação entre os grupos acidentados e não acidentados. Teste $t$ de Student para dados independentes. Teste do quiquadrado ou exato de Fisher.

O uso de equipamentos de proteção individual (EPIs) foi mais frequente $(97,5 \%)$ entre os profissionais acidentados, em comparação com os não acidentados (89,2\%), com diferença significativa $(p<0,0001)$. Os achados referentes aos tipos de 
EPIs utilizados estão detalhados no Gráfico.

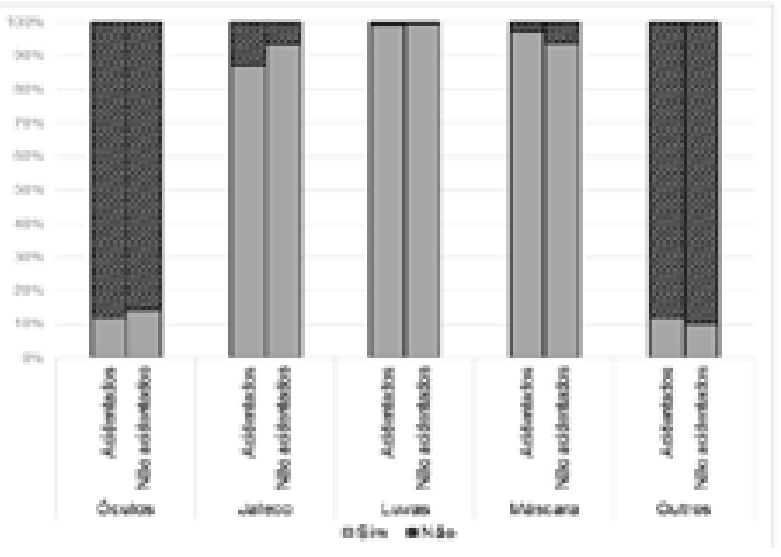

Gráfico. Distribuição dos 747 profissionais entrevistados conforme a utilização dos diferentes tipos de EPIs (equipamentos de proteção individual).

A análise por regressão logística mostrou que as variáveis sexo, categoria profissional e horário noturno apresentaram associação estatisticamente significante com a ocorrência de acidentes. A chance estimada de um profissional do sexo feminino sofrer acidente com instrumentos perfurocortantes foi quase três vezes maior que a de um profissional do sexo masculino (OR=2,67; IC95\%=1,82-3,91; $\mathrm{p}<0,0001)$. Os técnicos e os auxiliares de enfermagem apresentaram aproximadamente quatro a cinco vezes mais chance de se acidentar do que os enfermeiros (OR=3,88; IC95\%=2,276,64; $\mathrm{OR}=4,67 ; \quad \mathrm{IC} 95 \%=2,71-8,04)$. Profissionais que não trabalhavam em horário noturno apresentaram uma pequena chance a mais de sofrer esses acidentes em relação aos que trabalhavam à noite (OR=1,49; IC95\%=1,10-2,03). Quando questionados sobre o horário das ocorrências, a frequência de acidentes foi quase nove vezes maior no turno da manhã (360; $89,3 \%$ ), quando comparados à soma dos demais turnos (43; 10,6\%), e que mais da metade do total dos acidentes ocorreu no meio da jornada de trabalho (258; 64\%).

Embora a variável tempo de exercício profissional não tenha sido associada com a ocorrência de acidentes no modelo da regressão logística, foi observado que os técnicos e os auxiliares tinham em média 4,4l e 5,75 anos a mais na profissão, respectivamente, do que os enfermeiros, tendo sido a diferença entre as médias considerada estatisticamente significante $(p<0,0001)$.

A pele foi a superfície majoritariamente $(88,3 \%$; 356$)$ atingida nos acidentes por exposição aos fluidos biológicos, seguida da mucosa (6,7\%; 27$)$, pele e mucosa (2,2\%; 9), e não especificado $(2,8 \% ; 11)$. O sangue foi o fluido orgânico com o qual os profissionais tiveram mais contato na maioria dos acidentes (72\%; 290). Outros fluidos também mencionados foram: líquor $(2,5 \% ; 10)$, líquido pleural $(1,7 \% ; 7)$, líquido ascítico (2,2\%; 9), não especificado (21,6\%; 87). As agulhas foram responsáveis por $71 \%$ (286) dos acidentes produzidos pelos agentes perfurocortantes. Outros instrumentos mencionados foram: vidro (6,7\%; 27), tesoura (1\%; 4), lâmina de bisturi/lanceta (6,9\%; 28), ignorado (14,4\%; 58 ).

Grande parte dos profissionais atribuiu a ocorrência dos acidentes a fatores inerentes às condições de trabalho do hospital (espaço físico inadequado, uso de material inadequado). Esses fatores figuraram como quase um terço do total, e a segunda razão mais frequente, para a ocorrência dos acidentes, após a falta de atenção. A distribuição das circunstâncias dos acidentes mostrou que os procedimentos envolvendo a manipulação de agulhas (reencape de agulha, administração de medicamentos e punção venosa/arterial) somaram mais de metade das ocorrências (Tabela 3).

Tabela 3. Distribuição dos profissionais da enfermagem acidentados quanto à causa atribuída ao acidente e a circunstância da ocorrência ( $N$ = 403)

\begin{tabular}{|c|c|c|c|c|c|}
\hline VAstivets & rotal. & 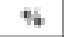 & VARLIVTE & $*(\mathbf{a})$ & sc 534 \\
\hline Cansa stribaids & & & Circenstieria & & \\
\hline 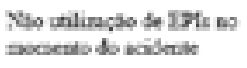 & 27 & 8.7 & Brececroanenso de aculta & $90(121)$ & $25.8 \cdot 3.7$ \\
\hline Iadigsicansso & $\$ 8$ & 14,4 & $\begin{array}{l}\text { Adminiversich dr } \\
\text { pediximetstos }\end{array}$ & $21 . t(85)$ & $12.1-8.1$ \\
\hline Falia be ascosta & 122 & toy & 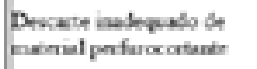 & $4,9(00)$ & $11,4 \cdot 18,6$ \\
\hline Elopse fivice iesdocwedo & 62 & 15.4 & 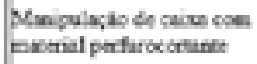 & $3.2(13)$ & 1.5-5. \\
\hline Liesparisis & 14 & 3,5 & 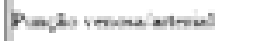 & 13,1 (53) & 9.9 .16 .6 \\
\hline Parede mupide & 5 & 12 & Dnogen atentrisis & $6.2(25)$ & $4,0.8,4$ \\
\hline $\begin{array}{l}\text { Une \& watcrial } \\
\text { iablequado }\end{array}$ & 56 & 139 & Ourros & 6.566 & $42-58$ \\
\hline 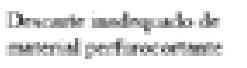 & 90 & 12,4 & Ifrarado & $s(x)$ & $3,0-6,9$ \\
\hline 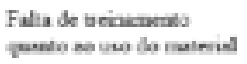 & 4 & 1,0 & & & \\
\hline Dustros & 5 & 12 & & & \\
\hline
\end{tabular}

\section{DISCUSSÃO}

No presente estudo foi encontrada uma frequência de $53,9 \%$ de acidentes por instrumentos perfurocortantes entre os profissionais de enfermagem expostos a fluidos biológicos no trabalho. Esse achado tem semelhança ao relatado, no Brasil, por outros autores ${ }^{(4,6)}$. Achados semelhantes foram também relatados por autores em países como Tailândia(7), Paquistão( ${ }^{(8)}$ e Quênia ${ }^{(9)}$, cuja frequência variou de 45,7 a 55,5\%. Tais achados parecem indicar que esse tipo de acidente tem se tornado um problema de saúde pública no mundo.

Ao lado da frequência de ocorrência desses acidentes, parece grave a situação relativa ao problema de sua subnotificação. Nos hospitais pesquisados, mais de um terço desses acidentes não têm sido notificados. Estudos acerca 
das razões para esta subnotificação pelos profissionais da saúde $^{(7,10)}$ relatam justificativas como a falta de tempo e o julgamento por parte do profissional de que o acidente não era grave. Desse modo é possivel inferir que esses profissionais não estejam conscientes do grande perigo que representa tal tipo de acidente, o que vem reforçar a necessidade das políticas de educação em saúde.

Um achado que podemos admitir como, ao menos, contraditário foi o de que os profissionais acidentados tinham uma maior idade, tempo de exercício profissional e de serviço nas instituições que trabalhavam. $O$ aumento da idade pode desencadear alterações cognitivas, como mudança no estado de alerta ou atenção, que adicionados a aspectos psicossociais como o estresse e a fadiga mental, podem levar ao sério comprometimento das condições de saúde e consequentemente de trabalho dos profissionais ${ }^{(11)}$. Um estudo em 2011 procurou compreender, na perspectiva dos profissionais de enfermagem, o significado dos acidentes de trabalho com exposição a material biológico. Os autores concluiram que a ocorrência de tais acidentes tem grande relação com a imprudência profissional, que pode estar relacionada à experiência e à prática adquirida durante vários anos de exercício profissional, que os leva a adquirirem uma postura de autoconfiança, não modificando comportamentos de risco mesmo quando reconhecem os perigos a que estão expostos $^{(12)}$. Corroborando esse fato, foi constatada que a subnotificação de acidentes foi significativamente maior entre os funcionários que tinham 10 anos ou mais de experiência na profissão(10).

Dos grupos profissionais de enfermagem que mais sofreram acidentes o destaque ficou para os auxiliares e técnicos de enfermagem. Este achado está em concordância com com relatado por diversos autores ${ }^{(2,13,14)}$. Esses autores justificaram seus achados destacando que: essas categorias representam a maior parcela de profissionais em uma equipe de enfermagem; o tipo de trabalho exercido requer a manipulação constante de materiais perfurocortantes; e o tempo maior de contato com os pacientes. 0 tipo de formação/educação oferecida para essas categorias pode também estar associada com o aumento do risco desse tipo de acidente ${ }^{(8)}$.

A maior ocorrência dos acidentes durante o turno de trabalho da manhã $(89,3 \%)$ pode estar relacionada ao fato de que este seja o horário de maior atividade dos hospitais. Isto pode ser atribuido a que o maior fluxo de pacientes demanda maior agilidade no atendimento, predispondo à ocorrência de acidentes por pressa, em detrimento da falta de atenção. Paradoxalmente os profissionais que trabalhavam à noite foram os que menos sofreram acidentes. Os próprios profissionais nomearam tais fatores como a principal causa de acidentes (30,3\%). Os fatores inerentes às condições de trabalho do hospital (espaço físico inadequado, uso de material inadequado), foram mencionados como a segunda maior causa (29,3\%). Dessa maneira, merece ser destacado que não basta apenas o profissional ter treinamento para execução dos procedimentos, mas também contar com as adequadas condições do seu local de trabalho, em acordo com as normas de segurança, para garantirem a prevenção dos acidentes.

Embora tenha sido significante a utilização de EPIs pelo grupo dos acidentados, foi constatado que o uso de tais equipamentos não evitaram, em boa parte dos profissionais, as lesões perfurocortantes, ocorridas usualmente após o rompimento de luvas.

A maior ocorrência de acidentes foi encontrada nos seguintes procedimentos: reencape de agulhas (30\%), administração de medicamentos (21,1\%), descarte inadequado de material perfurocortante e punção de vasos (13,15\%). Estes achados são diferentes dos relatados na literatura brasileira e estrangeira, em que foram relatados percentuais de 1,6 a $13 \%$ para o procedimento de reencape de agulhas ${ }^{(15-17)}$. Foram entretanto, semelhantes quanto ao descarte inadequado de materiais, relatados com percentuais de 10,16 a 12,9\% pelos mesmos autores. O reencape de agulhas é um hábito frequente entre os profissionais de saúde e, embora esteja associado com a ocorrência de acidentes, foi encontrado que um em cada cinco profissionais acreditava que reencapar agulhas era uma prática apropriada e segura(18).

$\mathrm{Na}$ Índia, foi relatado que, apesar de esforços educacionais regulares e intensivos, a maioria dos acidentes ocorreram quando os procedimentos padrões não foram seguidos, enquanto que em uma proporção muito menor ocorreram acidentes apesar de terem sido seguidas as precauções adequadas $^{(19)}$. Nos Emirados Árabes Unidos, foi relatado que o risco de sofrer uma lesão quase duplicou entre os profissionais que não estavam em conformidade com as precauções padrão (19\% contra $31 \%)$, dentre as quais incluíram a disponibilidade frequente de caixas de perfurocortantes e evitar reencapar agulhas e/ou retirá-las de seringas e após o uso(20). O conhecimento acerca de tais precauções exerce um grande impacto sobre a atitude do indivíduo com as mesmas, o que resultará em menos ocorrências como o acidente através de instrumentos perfurocortantes.

\section{Limitações do estudo}

Foi a não aplicação de medidas educativas e preventivas no período da realização do estudo na promoção, conscientização e capacitação dos profissionais da equipe de Enfermagem quanto às situações que representam risco ocupacional, tornando possivel a mudança de comportamento, bem como 
as subnotificações de acidentes de trabalho.

\section{Contribuições do estudo para prática}

Fornecer informações sobre os fatores de riscos de acidentes com materiais perfurocortantes, e levar uma proposta para as instituições para realização de campanhas no que diz respeito à prevenção de acidentes, notificação abrangente dos acidentes, acompanhamento dos trabalhadores que sofreram acidente, realização de capacitações quanto ao uso dos novos perfurocortantes e utilização dos dispositivos de segurança.

\section{CONCLUSÃO}

A exposição ocupacional a fluidos biológicos em acidentes com perfurocortantes na equipe de enfermagem hospitalar é alta. Há fatores inerentes às condições de trabalho dos profissionais que predispõem a um risco aumentado de acidentes. Por outro lado, posturas de risco tais como reencape de agulhas e descarte inadequado dos materiais perfurocortantes, ou seja, a não adesão às precauções básicas, também contribuem para a elevada ocorrência de acidentes. Essas práticas devem ser foco de políticas de educação em saúde, aliadas ao reforço da importância da notificação das ocorrências, uma vez que a subnotificação parece representar um dos principais obstáculos para o diagnóstico do problema.

\section{Contribuição dos autores}

Concepção e desenho, Análise e interpretação dos dados, Redação do artigo, Revisão crítica, Revisão final: Letícia Moreira Fontes, Iapunira Catarina Sant'Anna Aragão, Felipe Matheus Sant'Anna Aragão, Francisco Prado Reis, José Aderval Aragão. 


\section{REFERÊNCIAS}

1. Goniewicz M, Wtoszczak-Szubzda A, Niemcewicz M, Magdalena W, Marciniak-Niemcewicz A, Jarosz MJ. Injuries caused by sharp instruments among healthcare workers - international and Polish perspectives. Ann. Agric. Environ. Med. 2012; 19(3): 523-27. Available from: http://www.aaem.pl/Injuries-caused-by-sharp-instruments-among-healthcare-workers-international-and-Polish,71814,0,2.html

2. Barros DX, Tipple AFV, Lima LKOL, Souza ACS, Neves ZCP, Salgado TA. Análise de 10 anos de acidentes com material biológico entre a equipe de enfermagem. Rev. Eletr. Enf. 2016; 18(ell57): 1-1l. Available from: https://revistas.ufg.br/fen/article/view/35493/21259

3. Amaro Junior AS, Custódio JMO, Rodrigues VPS, Nascimento JMO. Risco biológico no contexto da prática de enfermagem: uma análise de situações favorecedoras. Rev Epidemiol Control Infect. 2015;5(1):42-6. Available from: https://online.unisc.br/ seer/index.php/epidemiologia/article/view/5396

4. Pimenta FR, Ferreira MD, Gir E, Hayashida M, Canini SRMS. Care and specialized clinical follow-up of nursing professionals who have been victims of accidents with biological material. Rev. Esc. Enferm. USP. 2013; 47(1): 198-204. Available from: http:// www.scielo.br/pdf/reeusp/v47nl/en_a25v47nl.pdf

5. Gomes AGM, Sabino TC, Negreiros RV. Acidentes de trabalho com materiais biológicos entre profissionais de enfermagem: uma revisão integrativa. Revista UninCor. 2016;14(2): 1119-27. Available from: http://periodicos.unincor.br/index.php/revistaunincor/article/view/2697/pdf_601

6. Machado MRM, Machado FA. Work-related accidents with biological material among nursing staff from General Hospital in Palmas, state of Tocantins, Brazil. Rev. bras. saúde ocup. 2011; 36(124): 274-81. Available from: http://www.scielo.br/pdf/rbso/ v36n124/allv36n124.pdf

7. Honda M, Chompikul J, Rattanapan C, Wood G, Klungboonkrong S. Sharps Injuries among Nurses in a Thai Regional Hospital: Prevalence and Risk Factors. Int. J. Occup. Environ. Med. 2011; 2(4): 215-23. Available from: http://www.theijoem.com/ijoem/index. php/ijoem/article/view/109/215

8. Janjua NZ, Khan MI, Mahmood B. Sharp injuries and their determinants among health care workers at first-level care facilities in Sindh Province, Pakistan. Trop. Med. Int. Health. 2010; 15(10): 244-51. Available from: https://onlinelibrary.wiley.com/ doi/epdf/10.1111/j.1365-3156.2010.02610.x

9. Mbaisi EM, Ng'ang'a Z, Wanzala P, Omolo J. Prevalence and factors associated with percutaneous injuries and splash exposures among health-care workers in a provincial hospital, Kenya, 2010. Pan African Medical Journal. 2013; 14:10. Available from: https:// www.ncbi.nlm.nih.gov/pmc/articles/PMC3597860/pdf/PAMJ14-10.pdf

10. Voidea C, Darling KEA, Kenfak-Foguena A, Erarda V, Cavassinia M, Lazor-Blanchet $C$. Underreporting of needlestick and sharps injuries among healthcare workers in a Swiss University Hospital. Swiss Med. Wkly. 2012; 142: 1-7. Available from: https:// smw.ch/article/doi/smw.2012.13523
11. Galon T, Robazzi MLCC, Marziale MHP. Occupational accidents with biological material at a university hospital in São Paulo. Rev. Eletr. Enf. 2008; 10(3): 673-85. Available from: http://www.scielo. $\mathrm{br} / \mathrm{pdf} / \mathrm{rlae} / \mathrm{v} 15 \mathrm{n} 4 / \mathrm{v} 15 \mathrm{n} 4 \mathrm{al7}$.pdf

12. Magagnini MAM, Rocha SA, Ayres JA. The meaning of accidents with biological material to nursing professionals. Rev. Gaúcha Enferm. 2011; 32(2):302-8. Available from: http://www.scielo. $\mathrm{br} / \mathrm{pdf} / \mathrm{rgenf} / \mathrm{v} 32 \mathrm{n} 2 / \mathrm{al3} 332 \mathrm{n} 2 . \mathrm{pdf}$

13. Valim M, Marziale M. Notification of work accidents with exposure to biological material: cross study. Online Brazilian Journal of Nursing. 2012; 11(1): 51-64. Available from: http://www.redalyc. org/pdf/3614/361441676006.pdf

14. Julio RS, Filardi MBS, Marziale MHP. Acidentes de trabalho com material biológico ocorridos em municipios de Minas Gerais. Rev. bras. enferm. 2014;67(1): 119-26. Available from: http://www. scielo.br/pdf/reben/v67n1/0034-7167-reben-67-01-0119.pdf

15. Noronha DD, Vieira MRM, Vieira MM, Magalhães TA, Leite MTS. Occupational accidents occurred between the professionals of the health of the University Hospital Clemente de Faria (UHCF). Motricidade. 2012; 8(2): 67-77. Available from: http://www.redalyc.org/html/2730/273023568009/index.html

16. Chaiwarith R, Ngamsrikam T, Fupinwong S, Sirisanthana T. Occupational Exposure to Blood and Body Fluids among Healthcare Workers in a Teaching Hospital: an Experience from Northern Thailand. 2013; Jpn. J. Infect. Dis. 66: 121-5. Available from: https://www.jstage.jst.go.jp/article/yoken/66/2/66_121/_pdf/-char/en

17. Frijstein G, Hortensius J, Zaaijer HL. Needlestick injuries and infectious patients in a major academic medical centre from 2003 to 2010. Neth. J. Med. 2011; 69(10): 465-8. Available from: http://www.njmonline.nl/getpdf.php?id=1115

18. Butsashvili M, Kamkamidze G, Kajaia M, Morse DL, Triner W, DeHovitz $\mathrm{J}$ et al. Occupational exposure to body fluids among health care workers in Georgia. Occupational Medicine. 2012; 62: 620-6. Available from: https://www.ncbi.nlm.nih.gov/pmc/articles/PMC3612004/pdf/kqs121.pdf

19. Jayanth ST, Kirupakaran H, Brahmadathan KN, Gnanaraj L, Kang G. Needle stick injuries in a tertiary care hospital. 2009; Indian J Med Microbiol. 27(1): 44-7. Available from: http://www. ijmm.org/article.asp?issn=2009; volume $=27$;issue $=1$;spage $=44 ; e^{-}$ page $=47$; aulast $=$ Jayanth

20. JacobA, Newson-Smith M, Murphy E, Steiner M, Dick F. Sharps injuries among health care workers in the United Arab Emirates. Occupational Medicine 2010; 60: 395-7. Available from: https:// www.ncbi.nlm.nih.gov/pubmed/20407045 